\title{
High-Resolution Wave Propagation Method for Stratified Flows
}

\author{
Nash'at N. Ahmad* \\ NASA Langley Research Center, Hampton, Virginia, 23681
}

\begin{abstract}
The implementation of the multidimensional $f$-waves Riemann solver for the timedependent, three-dimensional, nonhydrostatic, meso- and microscale atmospheric flows is described in detail. The Riemann solver employs flux-based wave decomposition ( $f$-waves) for the calculation of Godunov fluxes in which the flux differences are written directly as the linear combination of the right eigenvectors of the hyperbolic system. The scheme incorporates the source term due to gravity without introducing discretization errors which is an important property in the context of atmospheric flows. The resulting flow solver is conservative, accurate, stable, and well-balanced. The implementation of the solver is evaluated using benchmark test cases for atmospheric dynamics.
\end{abstract}

\section{Introduction}

molarkiewicz ${ }^{1}$ proposed his Multidimensional Positive Definite Advection Transport Algorithm (MPDATA) in $\checkmark$ the early eighties, which was adopted as the dynamical core of the Operational Multiscale Environment model with Grid Adaptivity (OMEGA). ${ }^{2}$ However, with the exception of the OMEGA model, the operational mesoscale atmospheric models have been based exclusively on finite difference schemes. ${ }^{3-7}$ In recent years, there has been a growing interest in using schemes other than finite-differences for atmospheric modeling. ${ }^{8-14}$ Substantial efforts have been made in evaluating finite-volume schemes for dynamical cores of atmospheric flow models. The FV3 (FiniteVolume3) ${ }^{13}$ developed by the Geophysical Fluid Dynamics Laboratory has recently been selected as the dynamical core of the National Oceanic and Atmospheric Administration's next generation models.

In this study, LeVeque's high-resolution wave propagation method ${ }^{15-19}$ is adapted for three-dimensional, timedependent atmospheric flows. The scheme employs flux-based wave decompositions ( $f$-waves) for the solution of the Riemann problem. The scheme is also well-balanced and incorporates the gravitational source term without introducing discretization errors. In the context of atmospheric flows, this is an important property. ${ }^{8,9,10,20}$ Ahmad and Lindeman have performed an extensive evaluation of the scheme in two dimensions using benchmark test cases. The scheme was also compared favorably with the Weather Research and Forecast (WRF) model.

Godunov-type schemes rely on the exact or approximate solution of the one-dimensional Riemann problem in each spatial direction for multidimensional problems. The shortcomings of this approach have been pointed out in the literature $^{21,22}$ and there is a vast body of literature on this topic going back to Roe's work in the 1980s. Loss of symmetry or spurious oscillations as a result of dimensional-splitting can degrade the accuracy of the solution. In some cases, it is possible to alleviate these problems by using a higher mesh resolution. Several multidimensional approaches have been suggested in the past, which include, for example: LeVeque's high-resolution wave propagation method, ${ }^{15}$ the corner transport upwind scheme, ${ }^{23}$ and the weighted average flux scheme. ${ }^{24}$ The $f$-waves solver developed for atmospheric flows in [9] was extended to three dimensions using dimensional-splitting in [25]. Although the three-dimensional $f$-waves solver with dimensional-splitting ${ }^{25}$ performed reasonably well on high-resolution grids, the loss of symmetry was observed, which in some cases led to inaccurate solutions on relatively coarse grids and larger integration times.

This paper describes in detail the development of the multidimensional $f$-waves Riemann solver for atmospheric flows on the meso- and microscales. In the following sections, the governing equations, the development of the Riemann solver for three-dimensional meso- and microscale atmospheric flows and its implementation within the Conservation Laws Package (CLAWPACK) ${ }^{26}$ - Version 4.1 is described in detail. Benchmark test cases are simulated to evaluate the performance of the scheme and also compared with results obtained using dimensional-splitting.

\footnotetext{
${ }^{*}$ Research Scientist, Computational Aerosciences Branch. Senior Member, AIAA.
} 


\section{Governing Equations}

The equations governing three dimensional atmospheric flows comprise a set of partial differential equations for the conservation of mass, momentum, and energy $\mathrm{y}^{2,27,28}$

$$
\frac{\partial \mathbf{U}}{\partial t}+\frac{\partial \mathbf{F}}{\partial x}+\frac{\partial \mathbf{G}}{\partial y}+\frac{\partial \mathbf{H}}{\partial z}=\mathbf{Q}+\mathbf{D}+\psi
$$

where,

$$
\mathbf{U}=\left[\begin{array}{c}
\rho \\
\rho u \\
\rho v \\
\rho w \\
\rho \theta
\end{array}\right], \quad \mathbf{F}=\left[\begin{array}{c}
\rho u \\
\rho u^{2}+p \\
\rho u v \\
\rho u w \\
u \rho \theta
\end{array}\right], \quad \mathbf{G}=\left[\begin{array}{c}
\rho v \\
\rho u v \\
\rho v^{2}+p \\
\rho v w \\
v \rho \theta
\end{array}\right], \quad \mathbf{H}=\left[\begin{array}{c}
\rho w \\
\rho u w \\
\rho v w \\
\rho w^{2}+p \\
w \rho \theta
\end{array}\right] .
$$

In Eq. (1)-(2), $\rho$ is the density of fluid, $u, v$, and $w$ are the velocity components, $p$ is the pressure and $\theta$ is the potential temperature. If a parcel of air at temperature $T$ and pressure $p$ is subjected to an adiabatic compression or expansion to a final reference pressure $\left(p_{0}\right)$, then its potential temperature, $\theta$ is given by:

$$
\theta=T\left(\frac{p_{0}}{p}\right)^{\frac{R_{d}}{c_{p}}} .
$$

The system is closed by an equation of state for pressure,

$$
p=C_{0}(\rho \theta)^{\gamma}
$$

where $C_{0}$ is a constant given by:

$$
C_{0}=\frac{R_{d}^{\gamma}}{p_{0}^{R_{d} / C_{v}}} .
$$

In the above relations, $\gamma\left(=C_{p} / C_{v}\right)$ is the ratio of specific heats, and $R_{d}$ is the gas constant for dry air. $C_{p}$ and $C_{v}$ are the specific heats of air at constant pressure and volume, respectively. In Eq. (1), $\mathbf{Q}$ is the source term due to microphysical processes of cloud formation and dissipation and atmospheric radiative transfer, and $\mathbf{D}$ is the diffusion term. For the purpose of this study, the atmosphere is assumed to be dry and the only source term is the gravitational force, $\psi$ acting in the vertical. With the exception of the density current benchmark, diffusion processes are also ignored and only the Euler solutions are considered. In the density current case, a constant eddy viscosity/diffusivity was used in both the momentum and potential temperature fields to obtain grid-converged solutions.

\section{Numerical Scheme}

The Riemann solver described in this paper has been implemented within the CLAWPACK software. CLAWPACK is an open-source software developed at the University of Washington, Seattle, by LeVeque. The user needs to provide a Riemann solver for the target hyperbolic partial differential equation, routines for model initialization, and source terms, if any. In this section, a brief description of the methodology implemented in CLAWPACK is given. The Euler equations (1)-(2) in one dimension can be written in the discrete form as:

$$
\mathrm{U}_{i}^{n+1}=\mathrm{U}_{i}^{n}-\Delta t\left[\frac{1}{\Delta z}\left(\mathrm{~F}_{i+\frac{1}{2}}-\mathrm{F}_{i-\frac{1}{2}}\right)\right],
$$


where $\mathrm{U}$ is the vector of conserved quantities, $\mathrm{F}$ is the vector of intercell fluxes calculated at the control surfaces of each control volume by using either an exact or approximate Riemann solver. $\Delta t$ and $\Delta z$ are the time step and spacing in $z$-direction, respectively. LeVeque ${ }^{15}$ and Bale et al. ${ }^{16}$ suggest using a flux-based wave decomposition, in which the flux differences $\mathrm{F}_{i}\left(\mathrm{U}_{i}\right)-\mathrm{F}_{i-1}\left(\mathrm{U}_{i-1}\right)$ are written directly as a linear combination of the right eigenvectors $r_{i-1 / 2}^{p}$,

$$
\mathrm{F}_{i}\left(\mathrm{U}_{i}\right)-\mathrm{F}_{i-1}\left(\mathrm{U}_{i-1}\right)-\Delta z \psi_{i-1 / 2}=\sum_{p=1}^{m} \beta_{i-1 / 2}^{p} r_{i-1 / 2}^{p} \equiv \sum_{p=1}^{m} Z_{i-1 / 2}^{p}
$$

and

$$
\beta_{i-1 / 2}=\mathrm{R}_{i-1 / 2}^{-1}\left(\mathrm{~F}_{i}\left(\mathrm{U}_{i}\right)-\mathrm{F}_{i-1}\left(\mathrm{U}_{i-1}\right)-\Delta z \psi_{i-1 / 2}\right)
$$

The vectors $Z^{p}=\beta^{p} r^{p}$ are called $f$-waves and contain flux increments rather than increments in $\mathrm{U} . \mathrm{R}_{i-1 / 2}$ is the matrix of right eigenvectors. $\psi=\rho g$, is the source term due to gravity - in the $x$ and $y$ directions, $\psi=0$. Eq. (6) can now be rewritten as:

$$
\mathrm{U}_{i}^{n+1}=\mathrm{U}_{i}^{n}-\frac{\Delta t}{\Delta z}\left[\mathcal{A}^{+} \Delta \mathrm{U}_{i-1 / 2}+\mathcal{A}^{-} \Delta \mathrm{U}_{i+1 / 2}\right]
$$

where

$$
\mathcal{A}^{-} \Delta \mathrm{U}_{i-1 / 2}=\sum_{p} Z_{i-1 / 2}^{p} \quad \text { if } \quad s_{i-1 / 2}^{p}<0, \text { and } \mathcal{A}^{+} \Delta \mathrm{U}_{i-1 / 2}=\sum_{p} Z_{i-1 / 2}^{p} \quad \text { if } \quad s_{i-1 / 2}^{p}>0
$$

The fluctuations $\mathcal{A}^{-} \Delta U_{i-1 / 2}$ and $\mathcal{A}^{+} \Delta U_{i-1 / 2}$ contribute to the cell-averaged quantity $\mathrm{U}_{i}$ due to the wave propagation across the cell interfaces. In the above relations, $s_{i-1 / 2}^{p}$ are the wave speeds given by the eigenvalues. Second-order accuracy is achieved by adding a correction term. ${ }^{15,18,19}$

$$
\mathrm{U}_{i}^{n+1}=\mathrm{U}_{i}^{n}-\frac{\Delta t}{\Delta z}\left[\mathcal{A}^{+} \Delta \mathrm{U}_{i-1 / 2}+\mathcal{A}^{-} \Delta \mathrm{U}_{i+1 / 2}\right]-\frac{\Delta t}{\Delta z}\left[\tilde{\mathrm{F}}_{i+1 / 2}-\tilde{\mathrm{F}}_{i-1 / 2}\right]
$$

where

$$
\tilde{\mathrm{F}}_{1-1 / 2}=\frac{1}{2} \sum_{p=1}^{m} \operatorname{sgn}\left(s_{i-1 / 2}^{p}\right)\left[1-\frac{\Delta t}{\Delta z}\left|s_{i-1 / 2}^{p}\right|\right] \tilde{Z}_{i-1 / 2}
$$

and, $\tilde{Z}^{p}$ is the limited value of $Z^{p}$. Given the $f$-waves and the wave speeds, the flux differences can be computed by summing up the left and right going waves across a control surface. In the above relations, the sweep in $z$-direction is implied. Similar methodology can be used for computations in the $x$ and $y$ directions without the addition of the gravitational source term. The quantities on cell faces are calculated by taking the average of cell-centered quantities on either side of the face. The development of the $f$-waves solver for atmospheric flows in two dimensions is described in detail by Ahmad and Lindeman ${ }^{9}$ and in three dimensions with dimensional-splitting in Ahmad. ${ }^{25}$

The algorithm can be extended for multidimensional problems by dimensional-splitting in which individual sweeps are performed in each spatial direction in succession. The unsplit extension of the algorithm ${ }^{15,18}$ takes into account contributions of wave fluctuations in the transverse direction. The solution of the Riemann problem in the transverse direction is analogous to the solution in the normal direction. The $\beta_{i}$ coefficients are calculated by replacing the jumps in flux functions, $\Delta F_{i}$ by the fluctuations $\mathcal{A}^{-} \Delta U_{i-1 / 2}$ and $\mathcal{A}^{+} \Delta U_{i-1 / 2}$. Implementation details of the multidimensional solver used in this study are given in Appendices $\mathrm{A}-\mathrm{C}$, and can be found in LeVeque ${ }^{15,18}$ for a general system of hyperbolic conservation laws. 


\section{Results}

The multidimensional $f$-waves implementation is evaluated using different benchmark cases in two and three dimensions - (1) Rayleigh-Taylor instability; ${ }^{29-30}$ (2) density current; ${ }^{31-32}$ (3) convection in neutral atmosphere; ${ }^{25,28}$ and (4) convection in stable atmosphere..$^{25,28}$

\section{A. Rayleigh-Taylor Instability}

Simulation of the Rayleigh-Taylor instability has been described previously in several publications. ${ }^{29-30}$ The setup in this section follows Almgren et al. ${ }^{29}$ The computational domain was defined by $(x, z) \in[0,0.5] \times[0,1] \mathrm{m}$ with $t \in[0,2.5] \mathrm{s}$. The mesh had a resolution of $\Delta x=\Delta z=0.00195 \mathrm{~m}(256 \times 512$ cells $)$. Periodic boundary conditions were used in the lateral, and the top and bottom boundaries were set to solid walls. The initial horizontal and vertical velocities were set to zero. The density was set to $\rho_{1}=1 \mathrm{~kg} / \mathrm{m}^{3}$ in the lower half of the domain and $\rho_{2}=2 \mathrm{~kg} / \mathrm{m}^{3}$ in the upper half of the domain. Pressure was initialized using the hydrostatic equation and a single-mode perturbation was introduced in density at the interface of heavier and lighter fluids.

The simulated density fields at time $=2.5 \mathrm{~s}$ are compared with the Almgren et al. ${ }^{29}$ results in Figure 1 . The top row shows the comparison of three different simulations with the $f$-waves solver using dimensional-splitting. Results obtained with the unsplit method are shown in the bottom row. Almgren et al. ${ }^{29}$ attribute the anomalies appearing in the solution (top row) to "alternating compression/expansion" due to operator-splitting. The oscillations also appear in the $f$-waves solution using dimensional-splitting but are not as pronounced.
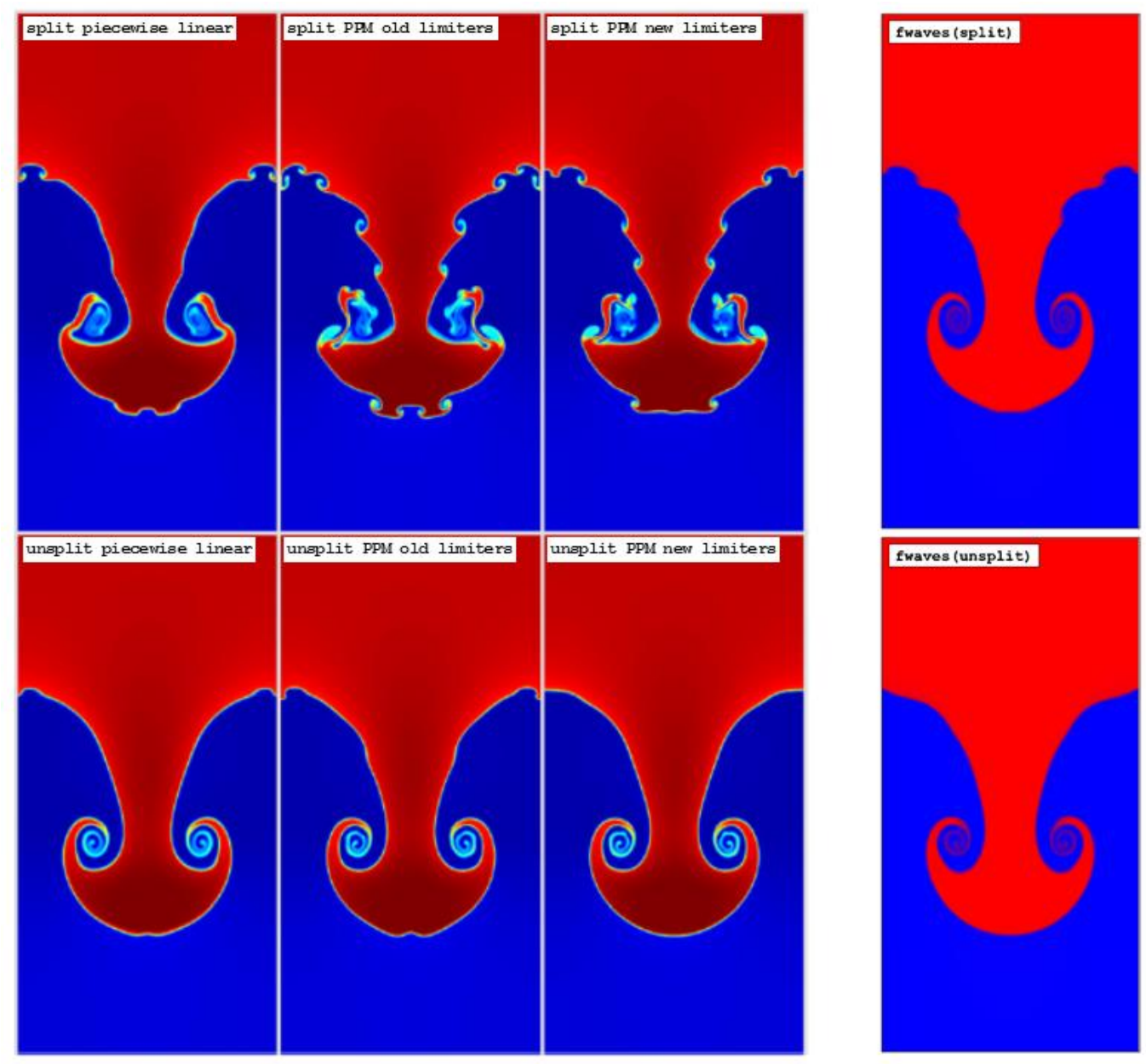

Figure 1. Rayleigh-Taylor Instability. The top row shows the comparison of density field with Almgren et al. ${ }^{29}$ at $2.5 \mathrm{~s}$ using dimensional-splitting. Results using the unsplit method at $2.5 \mathrm{~s}$ are shown in the bottom row. In the $f$-waves solution plots, the contour maximum is $2 \mathrm{~kg} / \mathrm{m}^{3}$ and the minimum is $1 \mathrm{~kg} / \mathrm{m}^{3}$. Number of contour levels was set to three. 


\section{B. Density Current}

The simulation of density current ${ }^{31-32}$ is described in this section. The computational domain was defined by $(x, z) \in[-25,25] \times[0,6.4] \mathrm{km}$ with $t \in[0,900] \mathrm{s}$. Simulations were run with uniform mesh spacing of $100 \mathrm{~m}$ and $25 \mathrm{~m}$. Outflow boundary conditions were used on the side boundaries and the top and the bottom boundaries were set to solid walls. The domain was initialized for a neutral atmosphere at $\theta_{0}=300 \mathrm{~K}$ in hydrostatic balance. The initial $u$ velocity and $w$-velocity were set to zero. Temperature profile in the vertical was defined by:

$$
T(z)=300-\frac{z g}{c_{p}}
$$

A cold bubble was initialized by adding a perturbation in the temperature field using the following relation:

$$
\Delta T= \begin{cases}0.0 & \text { if } L>1.0, \\ -15.0\left[\frac{\cos (\pi L)+1.0}{2}\right] & \text { if } L \leq 1.0\end{cases}
$$

where,

$$
L(x, z)=\sqrt{\left(\frac{x-x_{c}}{x_{r}}\right)^{2}+\left(\frac{z-z_{c}}{z_{r}}\right)^{2}}
$$

where, $\left(x_{c}, z_{c}\right)=(0,3) \mathrm{km}$ and $\left(x_{r}, z_{r}\right)=(4,2) \mathrm{km}$. A constant eddy viscosity $/$ diffusivity $\left(K_{\mathrm{m}}=K_{\mathrm{h}}=K=75 \mathrm{~m}^{2} / \mathrm{s}\right)$ was added in both the momentum and potential temperature fields.

This test case is an idealized simulation of a microburst that develops into a front. As the temperature perturbation is introduced into the domain, strong downdrafts are generated at the center of the cold bubble due to negative buoyancy. The cold air rolls up as it reaches the ground, and forms a front. Shear is generated at the top boundary of the front as it propagates forward. The benchmark solution ${ }^{31}$ consists of three rotors, which develop at the top boundary of the front due to a Kelvin-Helmholtz type instability. The comparison of the unsplit and the dimensionallysplit implementations at time $=900 \mathrm{~s}$ is given in Figure 2. In this test case, small differences can be observed on the coarse resolution grid $(\Delta x=\Delta z=100 \mathrm{~m})$, and the results are almost identical on the high-resolution grid $(\Delta x=\Delta z=$ $25 \mathrm{~m})$.
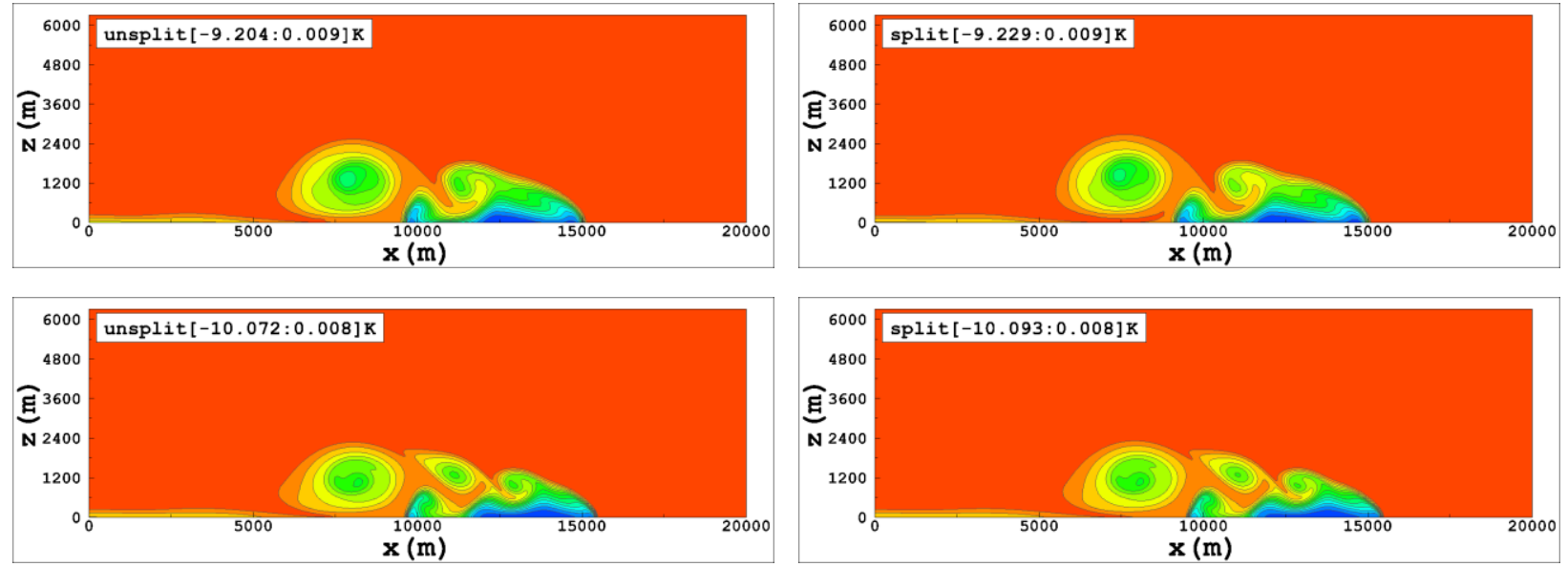

Figure 2. Density Current. The potential temperature perturbation field (K) at 900 s. Top row shows the comparison of split and unsplit simulations at $100 \mathrm{~m}$ resolution and the bottom row shows the solutions on the $25 \mathrm{~m}$ resolution grid. A colormap with fifteen contour levels and scaled by the domain maximum and minimum value was used. The domain maximum and minimum values are shown in each plot. 


\section{Convection in a Neutral Atmosphere}

Simulation of convection in a neutral atmosphere ${ }^{25,28}$ is described in this section. The computational domain for this case was defined by $(x, y, z) \in[0,4000] \times[0,4000] \times[0,4000] \mathrm{m}$ with $t \in[0,480] \mathrm{s}$. The mesh spacing was set to $\Delta x=\Delta y=\Delta z=80 \mathrm{~m}$. Outflow boundary conditions were used on the side boundaries, and the top and bottom boundaries were set to solid walls. The domain was initialized for a neutral atmosphere at $\theta_{0}=300 \mathrm{~K}$ in hydrostatic balance. The initial velocity $(u, v$, and $w)$ field was set to zero. A perturbation bubble in the potential temperature field was initialized at $x_{b}=x_{\max } / 2, y_{b}=y_{\max } / 2$, and $z_{b}=500 \mathrm{~m}$ :

$$
\theta(x, y, z)=\theta_{0}+\Delta \theta\left(1.0-\frac{L}{R}\right) \quad \text { if } \quad L \leq R,
$$

where,

$$
L=\sqrt{\left(x-x_{b}\right)^{2}+\left(y-y_{b}\right)^{2}+\left(z-z_{b}\right)^{2}} .
$$

The potential temperature perturbation maximum $\Delta \theta$, had a value of $2 \mathrm{~K}$. The radius $R$, of the perturbation was set to $500 \mathrm{~m}$.

The rising bubble test case is highly nonlinear and differences in solutions obtained using different numerical schemes are possible. The introduction of the thermal perturbation in the atmosphere generates large acceleration in the center of the bubble accompanied by downdrafts around the bubble core. Since, the temperature distribution inside the bubble is linear (highest temperature at the center), the center of the bubble rises faster. This creates sharp gradients of temperature in the upper part of the bubble. ${ }^{33}$

The comparison of the unsplit and dimensionally-split methods at time $=480 \mathrm{~s}$ are shown in Figure 3. The symmetry of the solution is preserved precisely in the unsplit implementation. In the dimensionally-split solution, there is loss of symmetry and the height of the bubble is not predicted correctly. Another set of simulations was performed with a uniform mesh resolution of $40 \mathrm{~m}$. The results are shown in Figures $4-5$. The dimensionally-split solution in the high resolution simulation is similar to the unsplit solution both in terms of the bubble shape and height. However, a slight loss in symmetry still exists in the solution.

\section{Convection in a Stable Atmosphere}

The computational domain was defined by $(x, y, z) \in[0,4000] \times[0,4000] \times[0,4000] \mathrm{m}$ with $t \in[0,480] \mathrm{s}$. The mesh spacing was set to $\Delta x=\Delta y=\Delta z=80 \mathrm{~m}$. Outflow boundary conditions were used on the side boundaries, and the top and bottom boundaries were set to solid walls. The domain was initialized for a stable atmosphere with a constant Brunt-Väisälä frequency, $N=10^{-2} \mathrm{~s}^{-1}$. The initial velocity field was set to zero. A potential temperature perturbation was initialized at $x_{b}=x_{\max } / 2, y_{b}=y_{\max } / 2$ and $z_{b}=500 \mathrm{~m}$ using Eq. (16)-(17). The potential temperature perturbation maximum $\Delta \theta$, had a value of $2 \mathrm{~K}$. The radius $R$, of the perturbation was set to $500 \mathrm{~m}$.

As the warm bubble rises through the stable atmosphere, it cools down due to the lower pressure at higher levels. The cooling of air parcels due to expansion, results in downdrafts in the bubble core. This is one of the mechanisms for the formation of thunderstorms. ${ }^{25}$

The comparison of the unsplit and dimensionally-split methods at time $=480 \mathrm{~s}$ are shown in Figure 6 . The symmetry of the solution is again preserved precisely in the unsplit implementation. In the dimensionally-split solution, there is a minor loss of symmetry, which is smaller compared to the neutral convection case. Another set of simulations was performed with a uniform mesh resolution of $40 \mathrm{~m}$. The results are shown in Figures 7-8. As in the previous case, both the dimensionally-split method and the unsplit method seem to be converging to a similar solution.

The figures also show that the gravity waves emanating from the sides of the updrafts do not create reflection problems at the outflow boundaries in both the split and the multidimensional implementation of the scheme. 

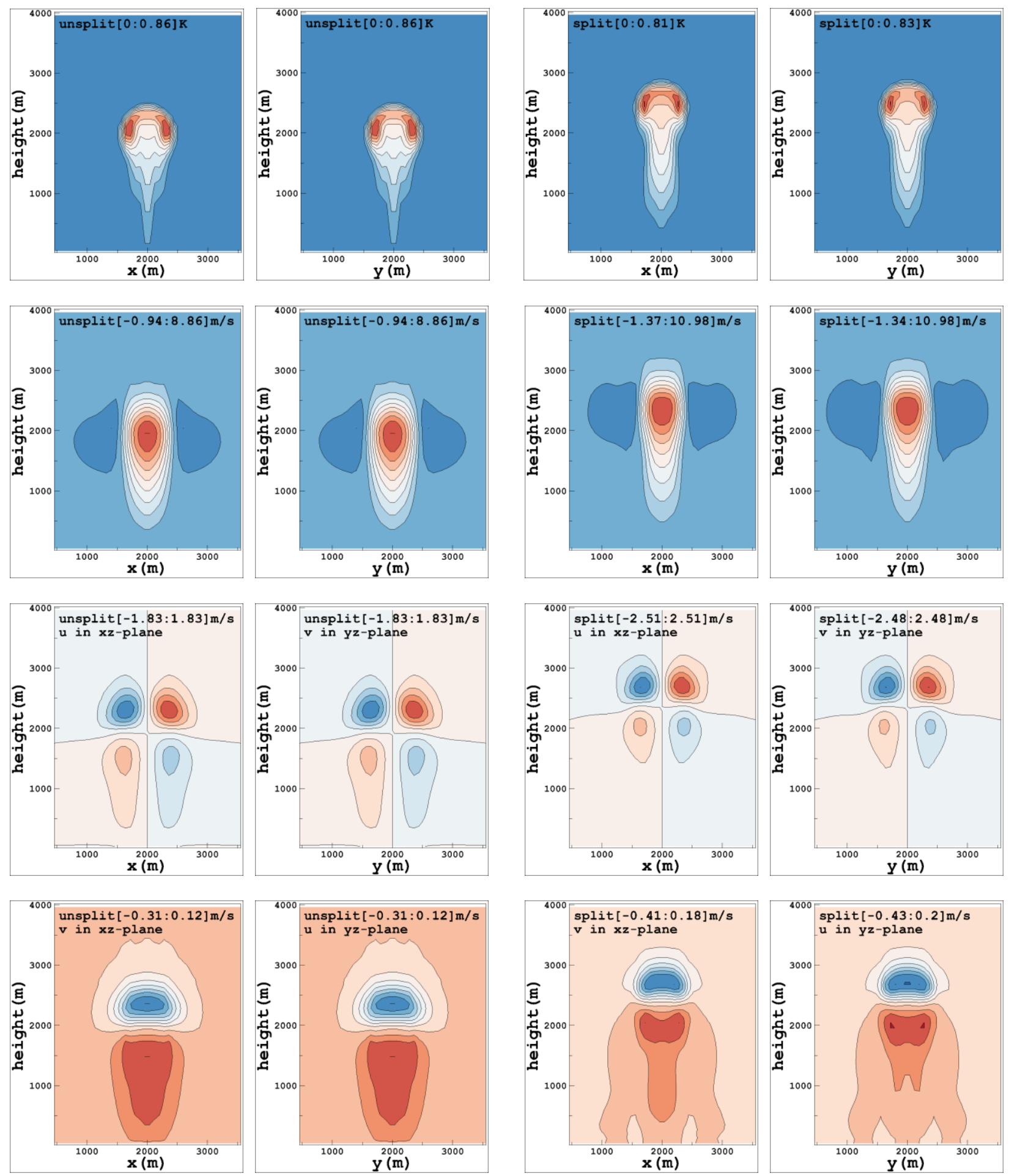

Figure 3. Convection in Neutral Atmosphere. Cross sections ( $x z$ at $y=y_{\max } / 2$ and $y z$ at $\left.x=x_{\max } / 2\right)$ of potential temperature perturbation (first row), vertical velocity (second row), and the horizontal velocity components (third and fourth rows) at time $=480 \mathrm{~s}$. The unsplit solutions are shown in the first two columns and with dimensional splitting in the two right columns. $\Delta x=\Delta y=\Delta z=80 \mathrm{~m}$. A colormap with eleven contour levels and scaled by the domain maximum and minimum values was used. The domain maximum and minimum values are shown in each plot. The symmetry of the solution is maintained precisely by the unsplit scheme. Large errors in the bubble height are seen in the dimensionally-split solution. 

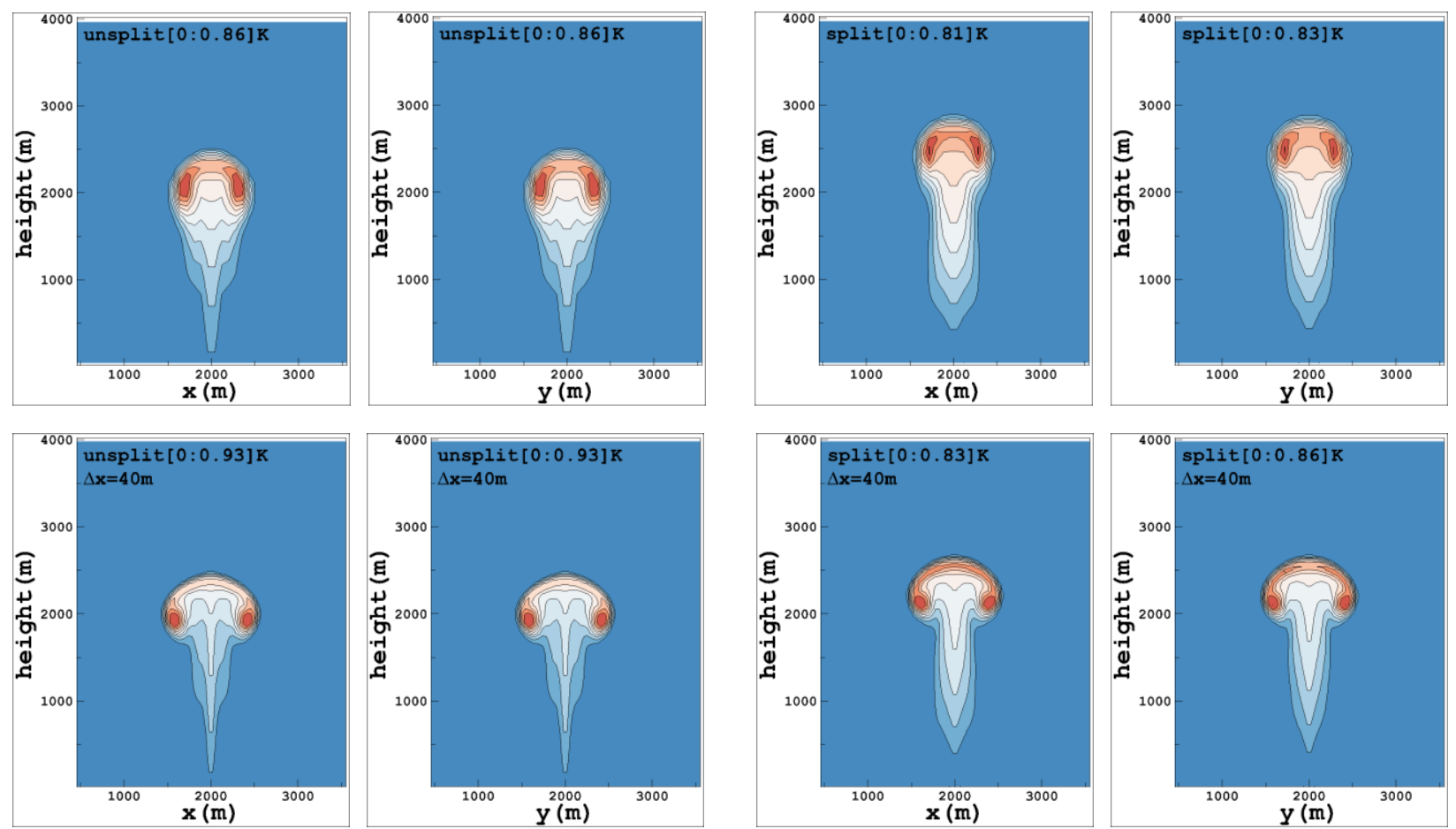

Figure 4. Convection in Neutral Atmosphere. Comparison of the potential temperature perturbation fields at different grid resolutions. $\Delta x=\Delta y=\Delta z=80 \mathrm{~m}$ (top row) and $\Delta x=\Delta y=\Delta z=40 \mathrm{~m}$ (bottom row). The number of contour levels is eleven and the plots are scaled by domain max/min values.
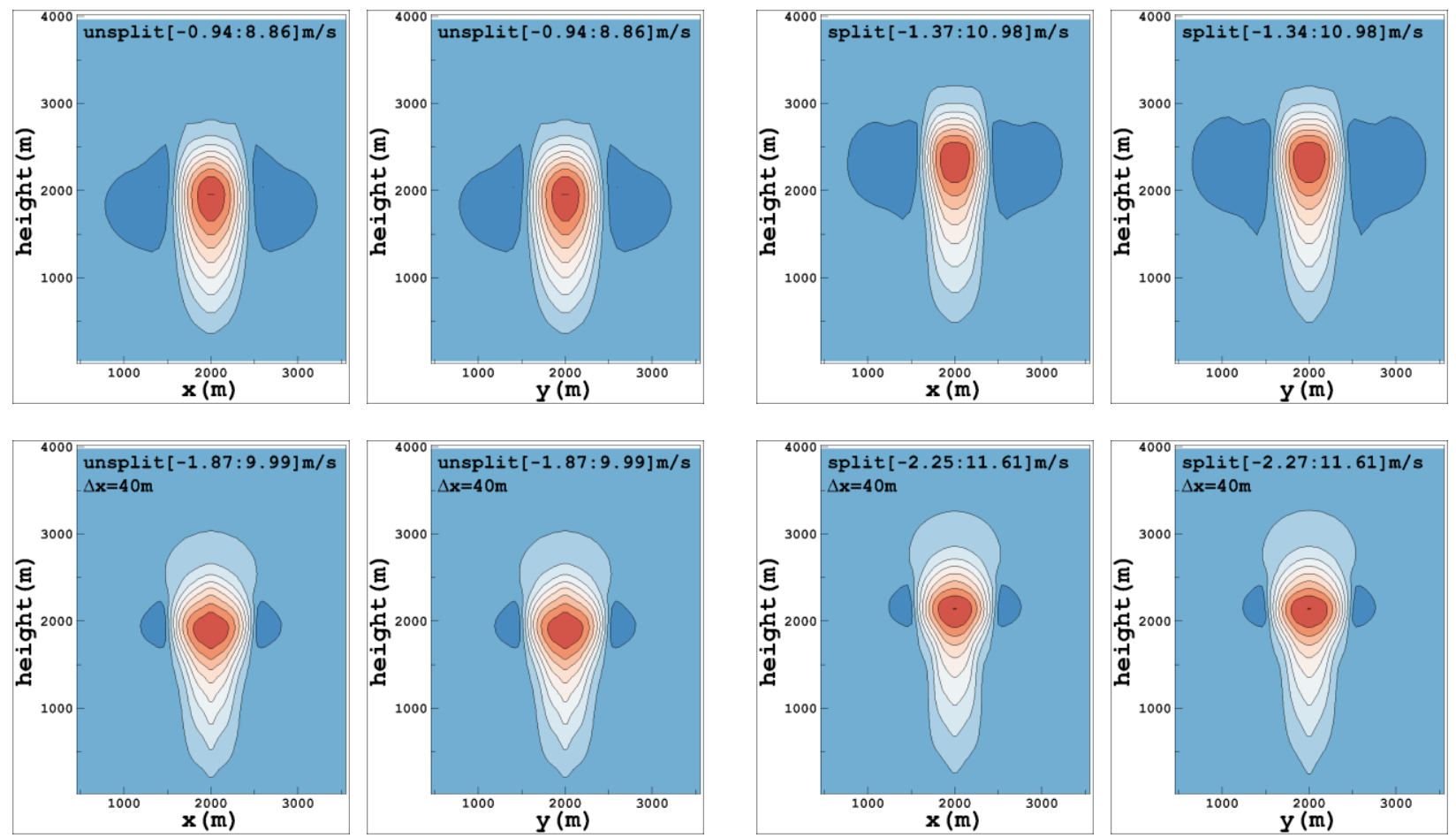

Figure 5. Convection in Neutral Atmosphere. Comparison of the vertical velocity fields at different grid resolutions. $\Delta x=\Delta y=\Delta z=80 \mathrm{~m}$ (top row) and $\Delta x=\Delta y=\Delta z=40 \mathrm{~m}$ (bottom row). The number of contour levels is eleven and the plots are scaled by domain max/min values. 

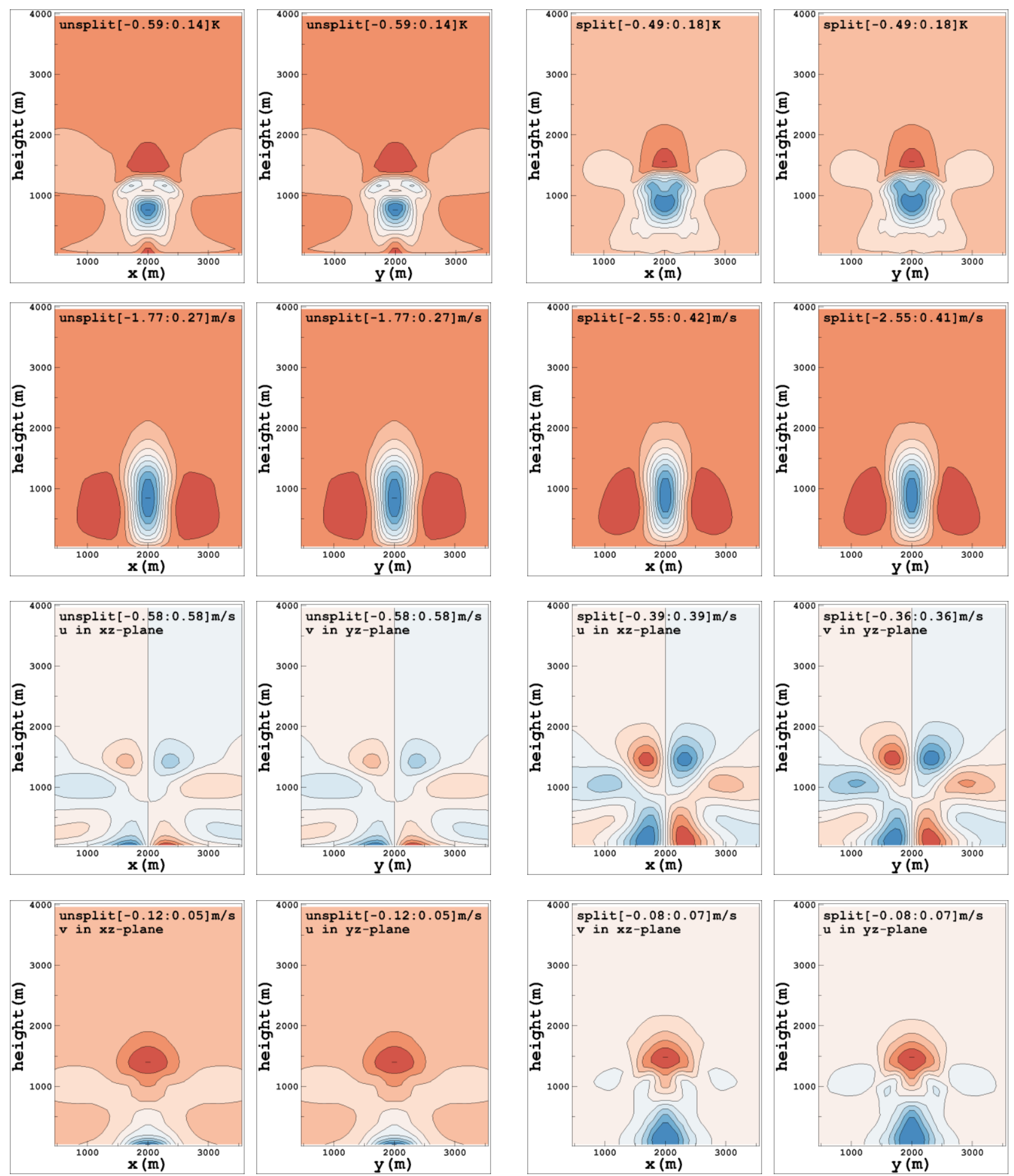

Figure 6. Convection in Stable Atmosphere. Cross sections $\left(x z\right.$ at $y=y_{\max } / 2$ and $y z$ at $\left.x=x_{\max } / 2\right)$ of potential temperature perturbation (first row), vertical velocity (second row), and the horizontal velocity components (third and fourth rows) at time $=480 \mathrm{~s}$. The unsplit solutions are shown in the first two columns and with dimensional splitting in the two right columns. $\Delta x=\Delta y=\Delta z=80 \mathrm{~m}$. A colormap with eleven contour levels and scaled by the domain maximum and minimum values was used. The domain maximum and minimum values are shown in each plot. The symmetry of the solution is maintained precisely by the unsplit scheme. 

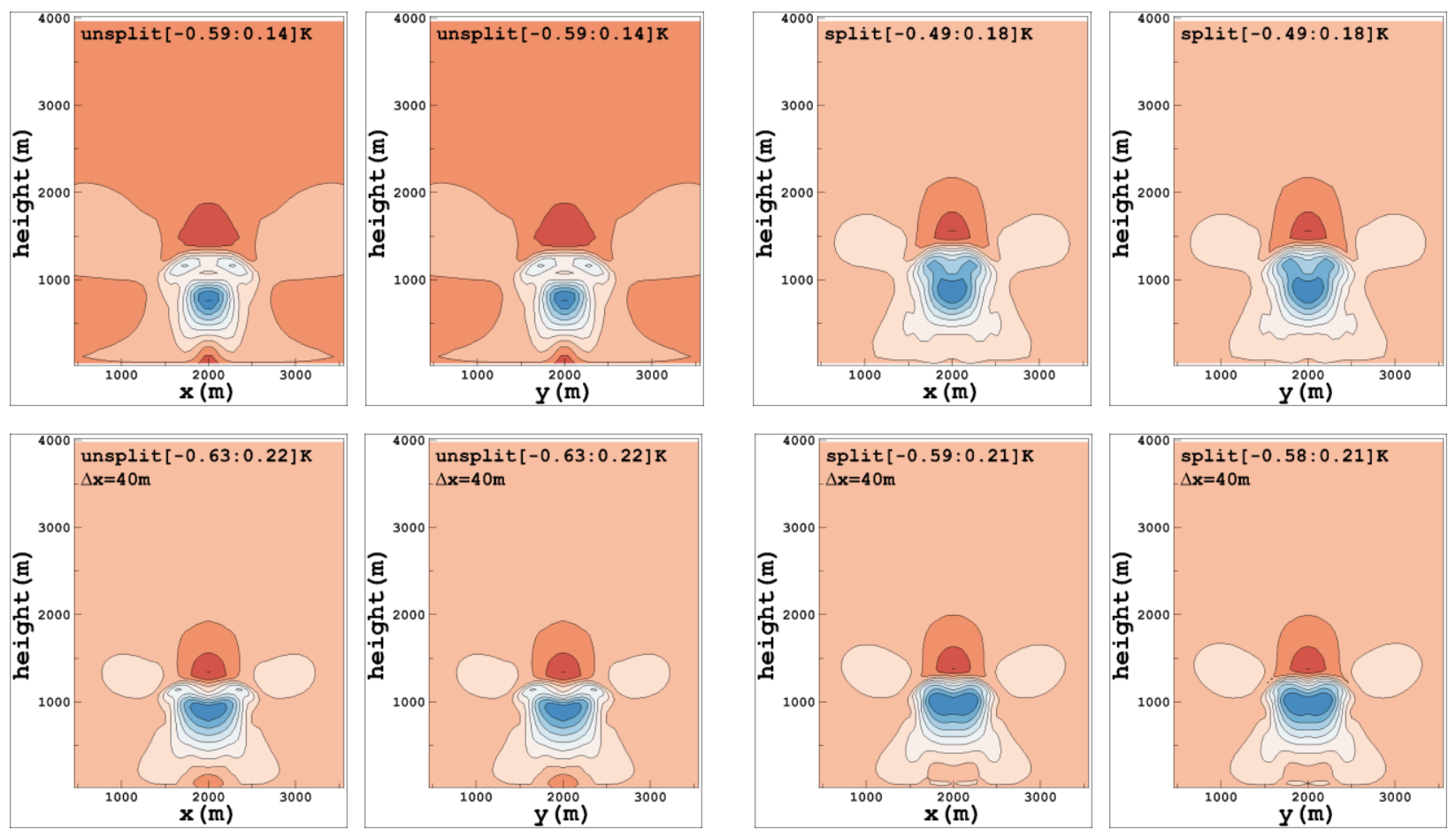

Figure 7. Convection in Stable Atmosphere. Comparison of the potential temperature perturbation fields at different grid resolutions. $\Delta x=\Delta y=\Delta z=80 \mathrm{~m}$ (top row) and $\Delta x=\Delta y=\Delta z=40 \mathrm{~m}$ (bottom row). The number of contour levels is eleven and the plots are scaled by domain max/min values.
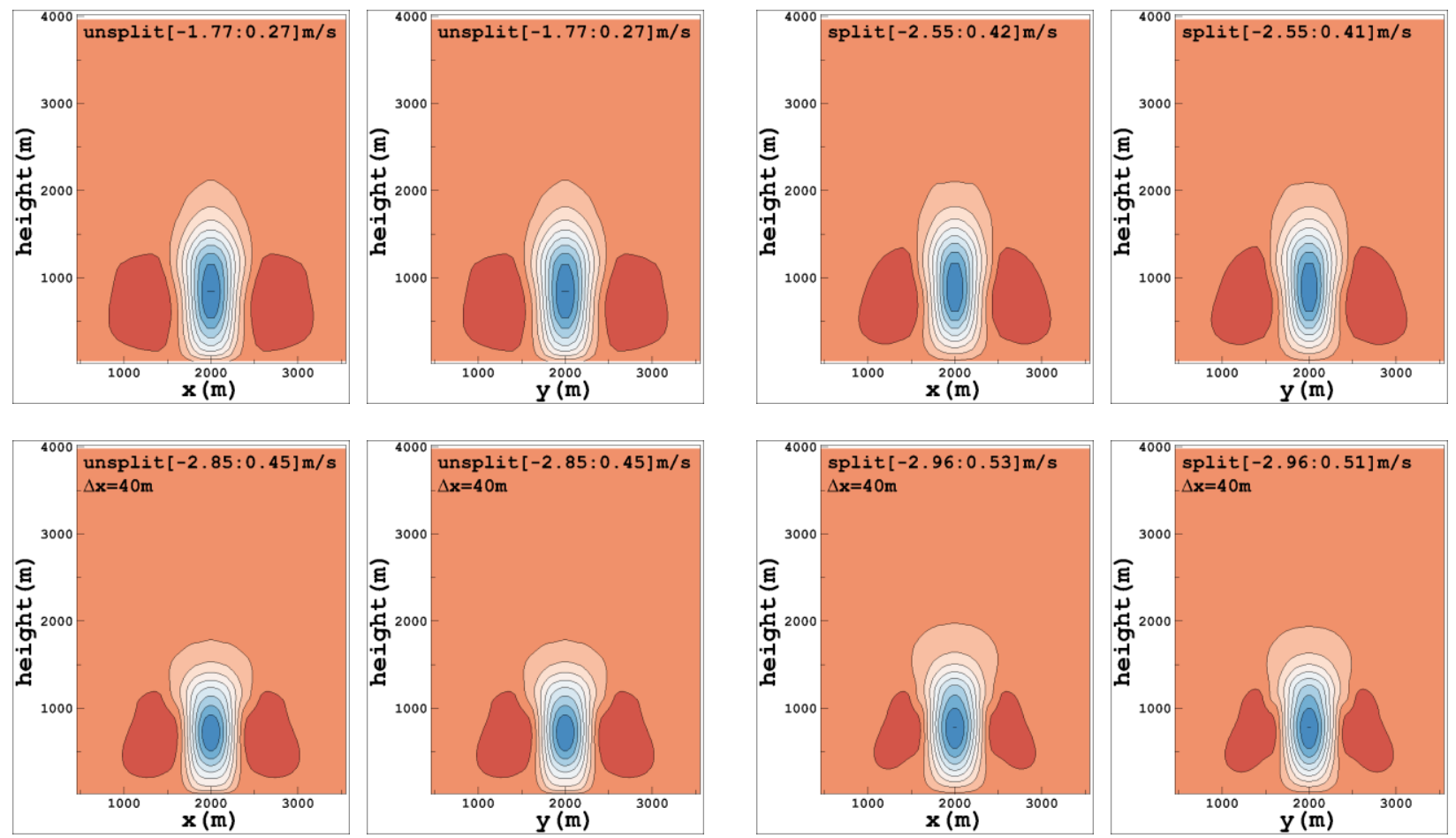

Figure 8. Convection in Stable Atmosphere. Comparison of the vertical velocity fields at different grid resolutions. $\Delta x=\Delta y=\Delta z=80 \mathrm{~m}$ (top row) and $\Delta x=\Delta y=\Delta z=40 \mathrm{~m}$ (bottom row). The number of contour levels is eleven and the plots are scaled by domain max/min values. 


\section{Summary}

A high-resolution, multidimensional Godunov-type scheme using the $f$-waves approximate Riemann solver after LeVeque was developed and successfully implemented for simulating atmospheric flows on the meso- and microscales in three dimensions. It was shown that the multidimensional implementation of the scheme is stable, accurate, and well-balanced. The symmetry of the solution is preserved precisely in the multidimensional implementation. In the dimensionally-split solution, there is some loss of symmetry. There was also a substantial degradation in accuracy in the case of convection in a neutral atmosphere. The loss of accuracy and symmetry in the dimensionally-split implementation was alleviated by increasing the mesh resolution. The three-dimensional simulations in this study were run to a final time of 480 s which is a relatively short time scale - further evaluations are needed to assess the performance of both schemes at larger integration times.

\section{Appendix A: Eigenstructure of the Euler Equations}

The eigenstructure of the three-dimensional Euler equations is needed for constructing the Riemann solver, and is presented in this appendix. The Euler equations can be written in quasi-linear form as:

$$
U_{t}+\mathbf{A}(U) U_{x}+\mathbf{B}(U) U_{y}+\mathbf{C}(U) U_{z}=0 .
$$

The subscripts $t, x, y$ and $z$ denote the derivatives in time and space. $\mathbf{A}(U), \mathbf{B}(U)$ and $\mathbf{C}(U)$ are the coefficient Jacobian matrices:

$$
\mathbf{A}(U)=\left[\begin{array}{ccccc}
0 & 1 & 0 & 0 & 0 \\
-u^{2} & 2 u & 0 & 0 & \frac{a^{2}}{\theta} \\
-u v & v & u & 0 & 0 \\
-u w & w & 0 & u & 0 \\
-u \theta & \theta & 0 & 0 & u
\end{array}\right], \mathbf{B}(U)=\left[\begin{array}{ccccc}
0 & 0 & 1 & 0 & 0 \\
-u v & v & u & 0 & 0 \\
-v^{2} & 0 & 2 v & 0 & \frac{a^{2}}{\theta} \\
-v w & 0 & w & v & 0 \\
-v \theta & 0 & \theta & 0 & v
\end{array}\right], \mathbf{C}(U)=\left[\begin{array}{ccccc}
0 & 0 & 0 & 1 & 0 \\
-u w & w & 0 & u & 0 \\
-v w & 0 & w & v & 0 \\
-w^{2} & 0 & 0 & 2 w & \frac{a^{2}}{\theta} \\
-w \theta & 0 & 0 & \theta & w
\end{array}\right],
$$

where, $a$ is the speed of sound. The eigenvalues and the eigenvectors corresponding to the Jacobian matrix $\mathbf{A}(U)$ in Eq. (A.2) are:

$$
u-a:\left[\begin{array}{c}
1 \\
u-a \\
v \\
w \\
\theta
\end{array}\right] \quad u:\left[\begin{array}{l}
0 \\
0 \\
1 \\
0 \\
0
\end{array}\right] \quad u:\left[\begin{array}{l}
0 \\
0 \\
0 \\
1 \\
0
\end{array}\right] \quad u:\left[\begin{array}{l}
1 \\
u \\
0 \\
0 \\
0
\end{array}\right] \quad u+a:\left[\begin{array}{c}
1 \\
u+a \\
v \\
w \\
\theta
\end{array}\right] .
$$

The matrix of right eigenvectors and its inverse are given by:

$$
R=\left[\begin{array}{ccccc}
1 & 0 & 0 & 1 & 1 \\
u-a & 0 & 0 & u & u+a \\
v & 1 & 0 & 0 & v \\
w & 0 & 1 & 0 & w \\
\theta & 0 & 0 & 0 & \theta
\end{array}\right] \text {, and } R^{-1}=\left[\begin{array}{ccccc}
\frac{u}{2 a} & -\frac{1}{2 a} & 0 & 0 & \frac{1}{2 \theta} \\
0 & 0 & 1 & 0 & -\frac{v}{\theta} \\
0 & 0 & 0 & 1 & -\frac{w}{\theta} \\
1 & 0 & 0 & 0 & -\frac{1}{\theta} \\
-\frac{u}{2 a} & \frac{1}{2 a} & 0 & 0 & \frac{1}{2 \theta}
\end{array}\right] \text {. }
$$


The eigenvalues and the eigenvectors corresponding to $\mathbf{B}(U)$ are:

$$
v-a:\left[\begin{array}{c}
1 \\
u \\
v-a \\
w \\
\theta
\end{array}\right] \quad v:\left[\begin{array}{l}
0 \\
1 \\
0 \\
0 \\
0
\end{array}\right] \quad v:\left[\begin{array}{l}
0 \\
0 \\
0 \\
1 \\
0
\end{array}\right] \quad v:\left[\begin{array}{l}
1 \\
0 \\
v \\
0 \\
0
\end{array}\right] \quad v+a:\left[\begin{array}{c}
1 \\
u \\
v+a \\
w \\
\theta
\end{array}\right]
$$

The matrix of right eigenvectors and its inverse are given by:

$$
R=\left[\begin{array}{ccccc}
1 & 0 & 0 & 1 & 1 \\
u & 1 & 0 & 0 & u \\
v-a & 0 & 0 & v & v+a \\
w & 0 & 1 & 0 & w \\
\theta & 0 & 0 & 0 & \theta
\end{array}\right], \text { and } R^{-1}=\left[\begin{array}{ccccc}
\frac{v}{2 a} & 0 & -\frac{1}{2 a} & 0 & \frac{1}{2 \theta} \\
0 & 1 & 0 & 0 & -\frac{u}{\theta} \\
0 & 0 & 0 & 1 & -\frac{w}{\theta} \\
1 & 0 & 0 & 0 & -\frac{1}{\theta} \\
-\frac{v}{2 a} & 0 & \frac{1}{2 a} & 0 & \frac{1}{2 \theta}
\end{array}\right] .
$$

The eigenvalues and the eigenvectors corresponding to $\mathbf{C}(U)$ are:

$$
w-a:\left[\begin{array}{c}
1 \\
u \\
v \\
w-a \\
\theta
\end{array}\right] \quad w:\left[\begin{array}{c}
0 \\
1 \\
0 \\
0 \\
0
\end{array}\right] \quad w:\left[\begin{array}{l}
0 \\
0 \\
1 \\
0 \\
0
\end{array}\right] \quad w:\left[\begin{array}{c}
1 \\
0 \\
0 \\
w \\
0
\end{array}\right] \quad w+a:\left[\begin{array}{c}
1 \\
u \\
v \\
w+a \\
\theta
\end{array}\right] .
$$

The matrix of right eigenvectors and its inverse are given by:

$$
R=\left[\begin{array}{ccccc}
1 & 0 & 0 & 1 & 1 \\
u & 1 & 0 & 0 & u \\
v & 0 & 1 & 0 & v \\
w-a & 0 & 0 & w & w+a \\
\theta & 0 & 0 & 0 & \theta
\end{array}\right], \text { and } R^{-1}=\left[\begin{array}{ccccc}
\frac{w}{2 a} & 0 & 0 & -\frac{1}{2 a} & \frac{1}{2 \theta} \\
0 & 1 & 0 & 0 & -\frac{u}{\theta} \\
0 & 0 & 1 & 0 & -\frac{v}{\theta} \\
1 & 0 & 0 & 0 & -\frac{1}{\theta} \\
-\frac{w}{2 a} & 0 & 0 & \frac{1}{2 a} & \frac{1}{2 \theta}
\end{array}\right] .
$$




\section{Appendix B: Riemann Solver in the Normal Direction}

The Riemann solver in the normal direction is described in detail in this appendix. For the dimensionally-split version of the solver, only this routine is called successively in all three directions.

1. In a loop over cell faces (subscript $i$ ), calculate $\rho, u, v, w, \theta$, and the speed of sound, $a$

$$
\begin{aligned}
& \rho_{i}=\frac{1}{2}\left(U_{1}^{l}+U_{1}^{r}\right) \\
& u_{i}=\frac{1}{2}\left(\frac{U_{m u}^{l}}{U_{1}^{l}}+\frac{U_{m u}^{r}}{U_{1}^{r}}\right) ; v_{i}=\frac{1}{2}\left(\frac{U_{m v}^{l}}{U_{1}^{l}}+\frac{U_{m v}^{r}}{U_{1}^{r}}\right) ; w_{i}=\frac{1}{2}\left(\frac{U_{m w}^{l}}{U_{1}^{l}}+\frac{U_{m w}^{r}}{U_{1}^{r}}\right) \\
& \theta_{i}=\frac{1}{2}\left(\frac{U_{5}^{l}}{U_{1}^{l}}+\frac{U_{5}^{r}}{U_{1}^{r}}\right) \\
& p_{i}=\frac{1}{2}\left[C_{0}\left(U_{5}^{l}\right)^{\gamma}+C_{0}\left(U_{5}^{r}\right)^{\gamma}\right] \\
& a_{i}=\sqrt{\frac{\gamma p_{i}}{\rho_{i}}}
\end{aligned}
$$

where, $m u=2, m v=3, m w=4$ for the sweep in $x$-direction; $m u=3, m v=4, m w=2$ for the sweep in $y$-direction; and $m u=4, m v=2, m w=3$ for the sweep in $z$-direction. Superscripts $l$, and $r$ denote the quantities to the left and the right of the cell interface respectively.

2. Calculate the quantities on the left and right side of the interface:

$$
\begin{aligned}
& \left(\rho_{i}^{l}, \rho_{i}^{r}\right)=\left(U_{1, i}^{l}, U_{1, i}^{r}\right) \\
& \left(u_{i}^{l}, u_{i}^{r}\right)=\left(\frac{U_{m u, i}^{l}}{U_{1, i}^{l}}, \frac{U_{m u, i}^{r}}{U_{1, i}^{r}}\right) \\
& \left(v_{i}^{l}, v_{i}^{r}\right)=\left(\frac{U_{m v, i}^{l}}{U_{1, i}^{l}}, \frac{U_{m v, i}^{r}}{U_{1, i}^{r}}\right) \\
& \left(w_{i}^{l}, w_{i}^{r}\right)=\left(\frac{U_{m w, i}^{l}}{U_{1, i}^{l}}, \frac{U_{m w, i}^{r}}{U_{1, i}^{r}}\right) \\
& \left(p_{i}^{l}, p_{i}^{r}\right)=\left(C_{0}\left(U_{5, i}^{l}\right)^{\gamma}, C_{0}\left(U_{5, i}^{r}\right)^{\gamma}\right)
\end{aligned}
$$

3. Calculate the jumps in the flux functions:

$$
\left[\begin{array}{c}
\Delta F_{1, i} \\
\Delta F_{2, i} \\
\Delta F_{3, i} \\
\Delta F_{4, i} \\
\Delta F_{5, i}
\end{array}\right]=\left[\begin{array}{c}
U_{m u, i}^{l}-U_{m u, i}^{r} \\
\left(\rho_{i}^{l} u_{i}^{l} u_{i}^{l}+p_{i}^{l}\right)-\left(\rho_{i}^{r} u_{i}^{r} u_{i}^{r}-p_{i}^{r}\right) \\
\rho_{i}^{l} u_{i}^{l} v_{i}^{l}-\rho_{i}^{r} u_{i}^{r} v_{i}^{r} \\
\rho_{i}^{l} u_{i}^{l} w_{i}^{l}-\rho_{i}^{r} u_{i}^{r} w_{i}^{r} \\
U_{5, i}^{l} u_{i}^{l}-U_{5, i}^{r} u_{i}^{r}
\end{array}\right]
$$

For the sweep in $z$-direction, add the gravitational source term:

$$
\Delta F_{2, i}=\Delta F_{2, i}+\frac{1}{2}\left(\rho_{i}^{l}+\rho_{i}^{r}\right) g \Delta z,
$$

where, $g$ is the acceleration due to gravity. 
4. The $\beta_{i}$ coefficients can now be calculated by multiplying the inverse of the matrix of right eigenvectors with the vector containing the jumps in fluxes:

$\left[\begin{array}{c}\beta_{1, i} \\ \beta_{2, i} \\ \beta_{3, i} \\ \beta_{4, i} \\ \beta_{5, i}\end{array}\right]=\left[\begin{array}{c}\frac{1}{2}\left(\frac{u_{i}}{a_{i}} \Delta F_{1, i}-\frac{1}{a_{i}} \Delta F_{2, i}+\frac{1}{\theta_{i}} \Delta F_{5, i}\right) \\ \Delta F_{3, i}-\frac{v_{i}}{\theta_{i}} \Delta F_{5, i} \\ \Delta F_{4, i}-\frac{w_{i}}{\theta_{i}} \Delta F_{5, i} \\ \Delta F_{1, i}-\frac{1}{\theta_{i}} \Delta F_{5, i} \\ \frac{1}{2}\left(-\frac{u_{i}}{a_{i}} \Delta F_{1, i}+\frac{1}{a_{i}} \Delta F_{2, i}+\frac{1}{\theta_{i}} \Delta F_{5, i}\right)\end{array}\right]$

5. Once the $\beta_{i}$ coefficients have been calculated, the $f$-waves, $Z^{p}=\beta^{p} r^{p}$ are computed as follows:

$$
\begin{aligned}
s_{i}^{1}=u_{i}-a_{i}:\left[\begin{array}{c}
Z_{1, i}^{1} \\
Z_{m u, i}^{1} \\
Z_{m v, i}^{1} \\
Z_{m w, i}^{1} \\
Z_{5, i}^{1}
\end{array}\right]=\left[\begin{array}{c}
\beta_{1, i} \\
\beta_{1, i}\left(u_{i}-a_{i}\right) \\
\beta_{1, i} v_{i} \\
\beta_{1, i} w_{i} \\
\beta_{1, i} \theta_{i}
\end{array}\right] ; s_{i}^{2}=u_{i}:\left[\begin{array}{c}
Z_{1, i}^{2} \\
Z_{m u, i}^{2} \\
Z_{m v, i}^{2} \\
Z_{m w, i}^{2} \\
Z_{5, i}^{2}
\end{array}\right]=\left[\begin{array}{c}
0 \\
0 \\
\beta_{2, i} \\
0 \\
0
\end{array}\right] ; s_{i}^{3}=u_{i}:\left[\begin{array}{c}
Z_{1, i}^{3} \\
Z_{m u, i}^{3} \\
Z_{m v, i}^{3} \\
Z_{m w, i}^{3} \\
Z_{5, i}^{3}
\end{array}\right]=\left[\begin{array}{c}
0 \\
0 \\
0 \\
\beta_{3, i} \\
0
\end{array}\right] ; s_{i}^{4}=u_{i}:\left[\begin{array}{c}
Z_{1, i}^{4} \\
Z_{m u, i}^{4} \\
Z_{m v, i}^{4} \\
Z_{m w, i}^{4} \\
Z_{5}^{4}
\end{array}\right]=\left[\begin{array}{c}
\beta_{4, i} \\
\beta_{4, i} u_{i} \\
0 \\
0 \\
0
\end{array}\right] ; \\
s_{i}^{5}=u_{i}+a_{i}:\left[\begin{array}{c}
Z_{1, i}^{5} \\
Z_{m u, i}^{5} \\
Z_{m v, i}^{5} \\
Z_{m w, i}^{5} \\
Z_{5, i}^{5}
\end{array}\right]=\left[\begin{array}{c}
\beta_{5, i} \\
\beta_{5, i}\left(u_{i}+a_{i}\right) \\
\beta_{5, i} v_{i} \\
\beta_{5, i} w_{i} \\
\beta_{5, i} \theta_{i}
\end{array}\right]
\end{aligned}
$$

6. Given the $f$-waves, $Z_{i}$ and the wave speeds, $s_{i}^{\text {mwave }}$, the flux differences are computed by summing up the left and right going waves across cell interfaces:

for meqn $=1$, equations

for $i=1$, faces

for mwave $=1$, waves

$$
\left(\mathcal{A}^{-} \Delta \mathrm{U}\right)_{\text {meqn }, i}=\left(\mathcal{A}^{-} \Delta \mathrm{U}\right)_{\text {meqn }, i}+Z_{\text {meqn }, i}^{\text {mwave }} \quad \text { if } \quad s_{i}^{\text {mwave }}<0
$$

else

$\left(\mathcal{A}^{+} \Delta \mathrm{U}\right)_{\text {meqn }, i}=\left(\mathcal{A}^{+} \Delta \mathrm{U}\right)_{\text {meqn }, i}+Z_{\text {meqn }, i}^{\text {mwave }}$ 


\section{Appendix C: Riemann Solver in the Transverse Direction}

The Riemann solver in the transverse direction is described in detail in this appendix. For splitting the fluctuations in a $y$-like direction, the $\beta_{i}$ coefficients, waves, and the wave speeds are calculated as follows:

$$
\left[\begin{array}{l}
\beta_{1, i} \\
\beta_{2, i} \\
\beta_{3, i} \\
\beta_{4, i} \\
\beta_{5, i}
\end{array}\right]=\left[\begin{array}{c}
\frac{1}{2}\left(\frac{v_{i}}{a_{i}}\left(\mathcal{A}^{ \pm} \Delta \mathrm{U}\right)_{1, i}-\frac{1}{a_{i}}\left(\mathcal{A}^{ \pm} \Delta \mathrm{U}\right)_{m v, i}+\frac{1}{\theta_{i}}\left(\mathcal{A}^{ \pm} \Delta \mathrm{U}\right)_{5, i}\right) \\
\left(\mathcal{A}^{ \pm} \Delta \mathrm{U}\right)_{m u, i}-\frac{u_{i}}{\theta_{i}}\left(\mathcal{A}^{ \pm} \Delta \mathrm{U}\right)_{5, i} \\
\left(\mathcal{A}^{ \pm} \Delta \mathrm{U}\right)_{m w, i}-\frac{w_{i}}{\theta_{i}}\left(\mathcal{A}^{ \pm} \Delta \mathrm{U}\right)_{5, i} \\
\left(\mathcal{A}^{ \pm} \Delta \mathrm{U}\right)_{1, i}-\frac{1}{\theta_{i}}\left(\mathcal{A}^{ \pm} \Delta \mathrm{U}\right)_{5, i} \\
\frac{1}{2}\left(-\frac{v_{i}}{a_{i}}\left(\mathcal{A}^{ \pm} \Delta \mathrm{U}\right)_{1, i}+\frac{1}{a_{i}}\left(\mathcal{A}^{ \pm} \Delta \mathrm{U}\right)_{m v, i}+\frac{1}{\theta_{i}}\left(\mathcal{A}^{ \pm} \Delta \mathrm{U}\right)_{5, i}\right)
\end{array}\right]
$$

where, $m u=2, m v=3, m w=4$ for the sweep in $x$-direction; $m u=3, m v=4, m w=2$ for the sweep in $y$-direction; and $m u=4, m v=2, m w=3$ for the sweep in $z$-direction.

$$
\begin{aligned}
& s_{i}^{1}=v_{i}-a_{i}:\left[\begin{array}{c}
Z_{1, i}^{1} \\
Z_{m u, i}^{1} \\
Z_{m v, i}^{1} \\
Z_{m w, i}^{1} \\
Z_{5, i}^{1}
\end{array}\right]=\left[\begin{array}{c}
\beta_{1, i} \\
\beta_{1, i} u_{i} \\
\beta_{1, i}\left(v_{i}-a_{i}\right) \\
\beta_{1, i} w_{i} \\
\beta_{1, i} \theta_{i}
\end{array}\right] ; s_{i}^{2}=v_{i}:\left[\begin{array}{c}
Z_{1, i}^{2} \\
Z_{m u, i}^{2} \\
Z_{m v, i}^{2} \\
Z_{m w, i}^{2} \\
Z_{5, i}^{2}
\end{array}\right]=\left[\begin{array}{c}
0 \\
\beta_{2, i} \\
0 \\
0 \\
0
\end{array}\right] ; s_{i}^{3}=v_{i}:\left[\begin{array}{c}
Z_{1, i}^{3} \\
Z_{m u, i}^{3} \\
Z_{m v, i}^{3} \\
Z_{m w, i}^{3} \\
Z_{5, i}^{3}
\end{array}\right]=\left[\begin{array}{c}
0 \\
0 \\
0 \\
\beta_{3, i} \\
0
\end{array}\right] ; s_{i}^{4}=v_{i}:\left[\begin{array}{c}
Z_{1, i}^{4} \\
Z_{m u, i}^{4} \\
Z_{m v, i}^{4} \\
Z_{m w, i}^{4} \\
Z_{5}^{4}
\end{array}\right]=\left[\begin{array}{c}
\beta_{4, i} \\
0 \\
\beta_{4, i} v_{i} \\
0 \\
0
\end{array}\right] ; \\
& s_{i, i}^{5}=v_{i}+a_{i}:\left[\begin{array}{c}
\beta_{5, i} \\
Z_{5, i}^{5} u_{i} \\
Z_{m v, i}^{5} \\
Z_{m w, i}^{5} \\
Z_{5, i}^{5}
\end{array}\right]=\left[\begin{array}{c}
\beta_{5, i}\left(v_{i}+a_{i}\right) \\
\beta_{5, i} w_{i} \\
\beta_{5, i} \theta_{i}
\end{array}\right]
\end{aligned}
$$

For splitting the fluctuations in a $z$-like direction, the $\beta_{i}$ coefficients, waves, and the wave speeds are calculated as follows:

$$
\left[\begin{array}{l}
\beta_{1, i} \\
\beta_{2, i} \\
\beta_{3, i} \\
\beta_{4, i} \\
\beta_{5, i}
\end{array}\right]=\left[\begin{array}{c}
\frac{1}{2}\left(\frac{w_{i}}{a_{i}}\left(\mathcal{A}^{ \pm} \Delta \mathrm{U}\right)_{1, i}-\frac{1}{a_{i}}\left(\mathcal{A}^{ \pm} \Delta \mathrm{U}\right)_{m w, i}+\frac{1}{\theta_{i}}\left(\mathcal{A}^{ \pm} \Delta \mathrm{U}\right)_{5, i}\right) \\
\left(\mathcal{A}^{ \pm} \Delta \mathrm{U}\right)_{m u, i}-\frac{u_{i}}{\theta_{i}}\left(\mathcal{A}^{ \pm} \Delta \mathrm{U}\right)_{5, i} \\
\left(\mathcal{A}^{ \pm} \Delta \mathrm{U}\right)_{m v, i}-\frac{v_{i}}{\theta_{i}}\left(\mathcal{A}^{ \pm} \Delta \mathrm{U}\right)_{5, i} \\
\left(\mathcal{A}^{ \pm} \Delta \mathrm{U}\right)_{1, i}-\frac{1}{\theta_{i}}\left(\mathcal{A}^{ \pm} \Delta \mathrm{U}\right)_{5, i} \\
\frac{1}{2}\left(-\frac{w_{i}}{a_{i}}\left(\mathcal{A}^{ \pm} \Delta \mathrm{U}\right)_{1, i}+\frac{1}{a_{i}}\left(\mathcal{A}^{ \pm} \Delta \mathrm{U}\right)_{m w, i}+\frac{1}{\theta_{i}}\left(\mathcal{A}^{ \pm} \Delta \mathrm{U}\right)_{5, i}\right)
\end{array}\right]
$$




$$
\begin{aligned}
& s_{i}^{1}=w_{i}-a_{i}:\left[\begin{array}{c}
Z_{1, i}^{1} \\
Z_{m, i, i}^{1} \\
Z_{m v, i}^{1} \\
Z_{m w, i}^{1} \\
Z_{5, i}^{1}
\end{array}\right]=\left[\begin{array}{c}
\beta_{1, i} \\
\beta_{1, i} u_{i} \\
\beta_{1, i} v_{i} \\
\beta_{1, i}\left(w_{i}-a_{i}\right) \\
\beta_{1, i} \theta_{i}
\end{array}\right] ; s_{i}^{2}=w_{i}:\left[\begin{array}{c}
Z_{1, i}^{2} \\
Z_{m u, i}^{2} \\
Z_{m v, i}^{2} \\
Z_{m w, i}^{2} \\
Z_{5, i}^{2}
\end{array}\right]=\left[\begin{array}{c}
0 \\
\beta_{2, i} \\
0 \\
0 \\
0
\end{array}\right] ; s_{i}^{3}=w_{i}:\left[\begin{array}{c}
Z_{1, i}^{3} \\
Z_{m u, i}^{3} \\
Z_{m v, i}^{3} \\
Z_{m w, i}^{3} \\
Z_{5, i}^{3}
\end{array}\right]=\left[\begin{array}{c}
0 \\
0 \\
\beta_{3, i} \\
0 \\
0
\end{array}\right] ; s_{i}^{4}=w_{i}:\left[\begin{array}{c}
Z_{1, i}^{4} \\
Z_{m u, i}^{4} \\
Z_{m v, i}^{4} \\
Z_{m w, i}^{4} \\
Z_{5}^{4}
\end{array}\right]=\left[\begin{array}{c}
\beta_{4, i} \\
0 \\
0 \\
\beta_{4, i} w_{i} \\
0
\end{array}\right] ; \\
& s_{i}^{5}=w_{i}+a_{i}:\left[\begin{array}{c}
Z_{1, i}^{5} \\
Z_{m u, i}^{5} \\
Z_{m v, i}^{5} \\
Z_{m w, i}^{5} \\
Z_{5, i}^{5}
\end{array}\right]=\left[\begin{array}{c}
\beta_{5, i} \\
\beta_{5, i} u_{i} \\
\beta_{5, i} v_{i} \\
\beta_{5, i}\left(w_{i}+a_{i}\right) \\
\beta_{5, i} \theta_{i}
\end{array}\right]
\end{aligned}
$$

The transverse fluctuations $\left(\mathcal{B}^{ \pm} \mathcal{A}^{ \pm} \Delta \mathrm{U}\right)_{i}$ can be calculated as follows:

for meqn $=1$, equations

$$
\begin{aligned}
\text { for } i= & 1, \text { faces } \\
\text { for mwave }=1 \text {, waves } & \\
& \left(\mathcal{B}^{-} \mathcal{A}^{ \pm} \Delta \mathrm{U}\right)_{\text {meqn }, i}=\left(\mathcal{B}^{-} \mathcal{A}^{ \pm} \Delta \mathrm{U}\right)_{\text {meqn }, i}+Z_{\text {meqn }, i}^{\text {mwave }} \text { if } s_{i}^{\text {mwave }}<0 \\
& \text { else } \\
& \left(\mathcal{B}^{+} \mathcal{A}^{ \pm} \Delta \mathrm{U}\right)_{\text {meqn }, i}=\left(\mathcal{B}^{+} \mathcal{A}^{ \pm} \Delta \mathrm{U}\right)_{\text {meqn, },}+Z_{\text {meqn }, i}^{\text {mwave }}
\end{aligned}
$$

The multidimensional version requires several calls to the Riemann solver in the transverse direction in all spatial directions, and can be as much as three times more expensive than the dimensionally-split version depending on the grid size.

\section{Acknowledgments}

The $f$-waves Riemann solver developed for the atmospheric flows was implemented within the CLAWPACK framework (Version 4.1). CLAWPACK has been developed by Prof. Randall J. LeVeque (University of Washington, Seattle).

\section{References}

${ }^{1}$ Smolarkiewicz, PK, “A Fully Multidimensional Positive Definite Advection Transport Algorithm with Small Implicit Diffusion,” Journal of Computational Physics, Vol. 54, 1984, pp. 325-362.

${ }^{2}$ Bacon, DP, NN Ahmad, Z Boybeyi, TJ Dunn, MS Hall, PCS Lee, RA Sarma, MD Turner, K Waight, S Young, and J Zack, “A Dynamically Adapting Weather and Dispersion Model: The Operational Multiscale Environment Model with Grid Adaptivity (OMEGA)," Monthly Weather Review, Vol. 128, 2000, pp. 2044-2076.

${ }^{3}$ Klemp, JB, WC Skamarock, and J Dudhia, "Conservative Split-Explicit Time Integration Methods for the Compressible Nonhydrostatic Equations,” Monthly Weather Review, Vol. 135, 2007, pp. 2897-2913.

${ }^{4}$ Xue, M, KK Droegemeier, and V Wong, "The Advanced Regional Prediction System (ARPS) - A Multi-scale Nonhydrostatic Atmospheric Simulation and Prediction Model. Part I: Model Dynamics and Verification," Meteorology and Atmospheric Physics, Vol. 75, 2000, pp. 161-193.

${ }^{5}$ Pielke, R, WR Cotton, RL Walko, CJ Tremback, WA Lyons, LD Grasso, ME Nicholls, “A Comprehensive Meteorological Modeling System - RAMS,” Meteorology and Atmospheric Physics, Vol. 49, 1992, pp. 69-91. 
${ }^{6}$ Hodur, RM, “The Naval Research Laboratory's Couples Ocean/Atmosphere Mesoscale Prediction System (COAMPS)," Monthly Weather Review, Vol. 125, 1997, pp. 1414-1430.

${ }^{7}$ Dudhia, J, “A Nonhydrostatic Version of the Penn State/NCAR Mesoscale Model: Validation Tests and Simulation of an Atlantic Cyclone and Cold Front," Monthly Weather Review, Vol. 121, 1993, pp. 1493-1513.

${ }^{8}$ Botta, N, R Klein, S Langenberg, and S Lutzenkirchen, "Well Balanced Finite Volume Methods for Nearly Hydrostatic Flows," Journal of Computational Physics, Vol. 196, 2004, pp. 539-565.

${ }^{9} \mathrm{Ahmad}, \mathrm{NN}$, and J Lindeman, “Euler Solutions using Flux-based Wave Decomposition," International Journal for Numerical Methods in Fluids, Vol. 54:1, 2007, pp. 41-72.

${ }^{10}$ Giraldo, FX, and M Restelli, “A Study of Spectral Element and Discontinuous Galerkin Methods for the Navier-Stokes Equations in Nonhydrostatic Mesoscale Atmospheric Modeling: Equations Sets and Test Cases," Journal of Computational Physics, Vol. 227, 2008, pp. 3849-3877.

${ }^{11}$ Marras, S, M Moragues, M Vázquez, O Jorba, G Houzeaux, “A Variational Multiscale Stabilized Finite Element Method for the Solution of the Euler Equations of Nonhydrostatic Stratified Flows,” Journal of Computational Physics, Vol. 236, 2013, pp. 380-407.

${ }^{12} \mathrm{Bao}, \mathrm{L}, \mathrm{R}$ Klöfkorn, and RD Nair, "Horizontally Explicit and Vertically Implicit (HEVI) Time Discretization Scheme for a Discontinuous Galerkin Nonhydrostatic Model,” Monthly Weather Review, Vol. 143, 2015, pp. 972-990.

${ }^{13}$ Lin, S, L Harris, X Chen, W Yao, and J Chai, "Colliding Modons: A Nonlinear Test for the Evaluation of Global Dynamical Cores,” Journal of Advances in Modeling Earth Systems, Vol. 9, 2017, pp. 2483-2492.

${ }^{14}$ Nazari, F, and RD Nair, “A Godunov-type Finite-Volume Solver for Nonhydrostatic Euler Equations with a Time-splitting Approach," Journal of Advances in Modeling Earth Systems, Vol. 9, 2017, pp. 1-17.

${ }^{15}$ LeVeque, RJ, "Finite Volume Methods for Hyperbolic Problems," Cambridge University Press, 2002.

${ }^{16}$ Bale, D, RJ LeVeque, S Mitran, and JA Rossmanith, “A Wave-propagation Method for Conservation Laws and Balance Laws with Spatially Varying Flux Functions,” SIAM Journal of Scientific Computing, Vol. 24, 2002, pp. 955-978.

${ }^{17}$ Ketcheson, DI, M Parsani, and RJ LeVeque, "High-order Wave Propagation Algorithms for Hyperbolic Systems," SIAM Journal of Scientific Computing, Vol. 35, 2013, pp. A351-A377.

${ }^{18}$ Langseth, JO, and RJ LeVeque, "A Wave-Propagation Method for Three-dimensional Hyperbolic Conservation Laws," Journal of Computational Physics, Vol. 165, 2000, pp. 126-166.

${ }^{19}$ LeVeque, RJ, "High-resolution Conservative Algorithms for Advection in Incompressible Flow," SIAM Journal of Numerical Analysis, Vol. 33, 1996, pp. 627-665.

${ }^{20}$ Ghosh, D, and EM Constantinescu, "Well-Balanced, Conservative Finite Difference Algorithm for Atmospheric Flows," AIAA Journal, Vol. 54, 2016, pp. 1370-1385.

${ }^{21}$ Roe, P, "Discrete Models for the Numerical Analysis of Time-dependent Multidimensional Gasdynamics," Journal of Computational Physics, Vol. 63, 1986, pp. 458-476.

${ }^{22}$ Van Leer, B, “Progress in Multi-dimensional Upwinding,” ICASE Report 92-43, 1992.

${ }^{23}$ Colella, P, "Multidimensional Upwind Methods for Hyperbolic Conservation Laws," Journal of Computational Physics, Vol. 87, 1990, pp. 171-200.

${ }^{24}$ Toro, E. F., "Riemann Solvers and Numerical Methods for Fluid Dynamics," Springer-Verlag, 1999.

${ }^{25}$ Ahmad, NN, “The $f$-wave Riemann Solver for Meso- and Micro-scale Flows,” AIAA Paper 2008-465.

${ }^{26}$ Clawpack Development Team, “Clawpack Version 4.1,” http://www.clawpack.org.

${ }^{27}$ Lilly, DK, “On the Numerical Simulation of Buoyant Convection,” Tellus, Vol. 14, 1962, pp. 148-172.

${ }^{28}$ Mendez-Nuñez, LR, and JJ Carroll, "Application of the MacCormack Scheme to Atmospheric Nonhydrostatic Models," Monthly Weather Review, Vol. 122. 1994, pp. 984-1000. 
${ }^{29}$ Almgren, AS, VE Beckner, JB Bell, MS Day, LH Howell, CC Joggerst, MJ Lijewski, A Nonaka, M Singer, and M Zingale, “CASTRO: A New Compressible Astrophysical Solver. I. Hydrodynamics and Self-Gravity,” The Astrophysical Journal, Vol. 715, 2010, pp. 1221-1238.

${ }^{30}$ Liska, R, and B Wendroff, "Comparison of Several Difference Schemes on 1D and 2D Test Problems for the Euler Equations," SIAM Journal of Scientific Computing, Vol. 25, 2003, pp. 995-1017.

${ }^{31}$ Straka, JM, RB Wilhelmson, LJ Wicker, JR Anderson, and KK Droegemeier, "Numerical Solutions of a Non-linear Density Current: A Benchmark Solution and Comparisons," International Journal for Numerical Methods in Fluids, Vol. 17, 1993, pp. 122.

${ }^{32}$ Janjić, ZI, JP Gerrity, and S Nickovic, “An Alternative Approach to Nonhydrostatic Modeling,” Monthly Weather Review, Vol. 129, 2001, pp. 1164-1178.

${ }^{33}$ Koračin, D, V Isakov and L Mendez-Nuñez, “A Cloud-resolving Model with the Radiation Scheme based on the Monte Carlo Method," Atmospheric Research, Vol. 47, 1998, pp. 437-459. 